\section{MMW Kommentar}

Die Kassenärztliche Bundesvereinigung (KBV) hatte die nunmehr in Kraft getretene Änderung zwar abgelehnt, da sie einen erheblichen Prüfaufwand für Vertragsärzte darstellt, konnte sich aber im G-BA nicht durchsetzen. Die Folge ist, dass bei Ausstellung einer Krankschreibung bei solchen Patienten nun das Erfragen der ausgeübten Tätigkeit sowie der durchgeführten oder beabsichtigten Wiedereingliederungsmaßnahmen gehört. Die Entscheidung des Arztes hat dabei für die Patienten unmittelbar leistungsrechtliche Konsequenzen, da die Arbeitsunfähigkeitsbescheinigung der Bundesagentur für Arbeit in erster Linie zur Prüfung von Leistungsansprüchen der ALG II-Empfänger dient.

Niedergelassene Ärzte werden hier praktisch gezwungen, kostenlos bei solchen Patienten die Funktion des Medizinischen Dienstes (MDK) zu übernehmen. Wertvolle Arbeitszeit im Sprechzimmer, die eigentlich wirklich behandlungsbedürftigen Patienten zugute kommen sollte, wird deshalb in der Zukunft durch Diskussionen um eine solche Maßnahme verloren gehen. Schlimmer noch: das Bundesministerium für Gesundheit (BMG) hat den Beschluss des G-BA nicht beanstandet, sondern mit einer Auflage versehen. So muss der G-BA den in §2 Absatz 3 Satz 1 der AU-RL verwendeten Begriff „Bezieher von Arbeitslosengeld“ bis zum 31. März 2013 anpassen. Die Vorschrift muss dann auch noch Personen umfassen, die nur aufgrund einer Urlaubsabgeltung oder einer Sperrzeit kein Arbeitslosengeld beziehen oder über deren Arbeitslosengeld-Antrag noch nicht entschieden wurde.

\title{
Kompressionsverband abrechnen
}

— Verbände sind in der Regel Bestandteil der hausärztlichen Versichertenpauschale. Eine Ausnahme stellt die Nr. 02313 EBM dar, hinter der sich auch der Kompressionsverband verbirgt. Voraussetzung für die Abrechnung der Leistung ist aber die Dokumentation des Beinumfanges an mindestens drei Messpunkten und das Vorliegen mindestens der Diagnosen chronisch venöse Insuffizienz, postthrombotisches Syndrom, tiefe Beinvenenthrombose, oberflächliche Beinvenenthrombose und/oder Lymphödem.

Aber nicht nur das Anlegen eines Kompressionsverbandes ist nach Nr. 02313 EBM berechnungsfähig. Der Leistungsinhalt ist schon erfüllt, wenn zum Beispiel ein pati- entenindividueller Kompressionsstrumpf angelegt wurde und die geforderte Dokumentation des Beinumfanges zu den angegebenen Zeitpunkten erfolgte. Die früher geforderte Fotodokumentation ist nicht mehr erforderlich.

\section{MMW Kommentar}

Die Nr. 02313 EBM unterliegt im Quartal einer Höchstpunktzahl von 12000 Punkten. Der Höchstwert ist dabei auch auf den Arztfall anzuwenden, $d . h$. in einer Zweiergemeinschaftspraxis verdoppelt sich diese Punktzahl. Die Nr. 02313 EBM ist im hausärztlichen Bereich nicht neben den Nrn. 02310, 02311, 02350 und 30501 EBM berechnungsfähig.

\section{Hyposensibilisierung ist auch eine Leistung für Hausärzte}

— Die Leistung nach Nr. 30130 EBM (Hyposensibilisierungsbehandlung, 265 Punkte) kann nur einmal am Behandlungstag berechnet werden. Seit dem 1. Januar 2012 gibt es allerdings einen Zuschlag für jede weitere Hyposensibilisierungsbehandlung. Dieser Zuschlag nach Nr. 30131 EBM kann für jede weitere Hyposensibilisierungsbe- handlung durch Injektion(en) zu unterschiedlichen Zeiten am selben Behandlungstag bis zu viermal neben der Nr. 30130 EBM berechnet werden. Bei der Mehrfachberechnung der Nr. 30131 EBM bzw. der Nebeneinanderberechnung der Nrn. 30130 und 30131 müssen jeweils Uhrzeitangaben gemacht werden.

\section{MMW Kommentar}

Die Gebührenordnungspositionen des $A b$ schnitts 30.1 Allergologie können nur von Fachärzten für Hals-Nasen-Ohrenheilkunde, Fachärzten für Haut- und Geschlechtskrankheiten, Fachärzten für Innere Medizin mit Schwerpunkt Pneumologie und Lungenärzten sowie Fachärzten für Kinder- und Jugendmedizin berechnet werden. Hausärzte müssen als Abrechnungsvoraussetzung die Zusatzbezeichnung Allergologie tragen. Diese Einschränkung gilt allerdings nicht für die Gebührenordnungspositionen nach den
Nrn. 30130 und 30131 EBM, die von allen Vertragsärzten - soweit dies berufsrechtlich zulässig ist - berechnet werden können. Beachtenswert ist dabei, dass nach Ausführung der Leistung eine Nachbeobachtung von mindestens 30 Minuten Dauer gefordert ist und für den Fall einer anaphylaktischen Reaktion die Voraussetzungen für die notwendigen, sachlichen und personellen Bedingungen für eine gegebenenfalls erforderliche Schockbehandlung und Intubation vorhanden sein müssen. 